\title{
Predicting Group Contribution Behaviour in a Public Goods Game from Face-to-Face Communication
}

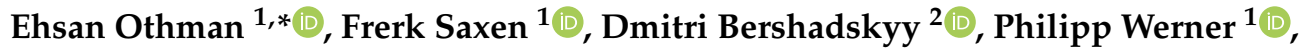 \\ Ayoub Al-Hamadi ${ }^{1}$ and Joachim Weimann ${ }^{3}$ (D) \\ 1 Neuro-Information Technology group, Institute for Information Technology and Communications, \\ Otto-von-Guericke University Magdeburg, 39106 Magdeburg, Germany; Frerk.Saxen@ovgu.de (F.S.); \\ Philipp.Werner@ovgu.de (P.W.); Ayoub.Al-Hamadi@ovgu.de (A.A.-H.) \\ 2 Halle Institute for Economic Research, 06108 Halle, Germany; Dmitri.Bershadskyy@iwh-halle.de \\ 3 Faculty of Economics and Management, Otto-von-Guericke University Magdeburg, 39106 Magdeburg, \\ Germany; Joachim.Weimann@ovgu.de \\ * Correspondence: Ehsan.Othman@ovgu.de
}

Received: 9 May 2019; Accepted: 17 June 2019; Published: 21 June 2019

\begin{abstract}
Experimental economic laboratories run many studies to test theoretical predictions with actual human behaviour, including public goods games. With this experiment, participants in a group have the option to invest money in a public account or to keep it. All the invested money is multiplied and then evenly distributed. This structure incentivizes free riding, resulting in contributions to the public goods declining over time. Face-to-face Communication (FFC) diminishes free riding and thus positively affects contribution behaviour, but the question of how has remained mostly unknown. In this paper, we investigate two communication channels, aiming to explain what promotes cooperation and discourages free riding. Firstly, the facial expressions of the group in the 3-minute FFC videos are automatically analysed to predict the group behaviour towards the end of the game. The proposed automatic facial expressions analysis approach uses a new group activity descriptor and utilises random forest classification. Secondly, the contents of FFC are investigated by categorising strategy-relevant topics and using meta-data. The results show that it is possible to predict whether the group will fully contribute to the end of the games based on facial expression data from three minutes of FFC, but deeper understanding requires a larger dataset. Facial expression analysis and content analysis found that FFC and talking until the very end had a significant, positive effect on the contributions.
\end{abstract}

Keywords: face-to-face communications (FFC); voluntary contribution mechanism (VCM); random forest classification $(\mathrm{RFc})$; facial activity descriptors (FADs); group activity descriptors (GADs); public goods game

\section{Introduction}

Cooperation is one of the major foundations of economic actions. Given its essential role, a lot of research in economics focuses on how cooperation can be improved. At the heart of this research is the evidence that communication is a highly effective tool to mediate social dilemmas [1]. A very straightforward way to analyse social dilemmas in experimental economics research is to use the public goods game, an experimental setup that allows for cooperation and incentivises free riding. However, the main research goal is to sustain cooperation and stop free riding [2]. An especially non-intrusive and simultaneously very effective way of doing this is to provide the participants of the experiment with an opportunity to communicate with each other prior to the public goods game itself. This free-form, face-to-face communication (FFC) induces group members to make more socially 
optimal contributions in public goods games independent of whether the FFC takes place in person or in a video-conference [3]. In this context, we introduce the following issue to the computer vision and behaviour understanding domain: the prediction and understanding of (future) behaviour based on FFC analysis. We automatically analyse the group's FFC in a laboratory public goods experiment with the goal of explaining if (and how) non-verbal communication enables the prediction of the group's future behaviour.

In this paper, we predict the end-game contribution behaviour of groups in a public goods game [4] after a 3-minute video-based communication using automatic facial expression analysis. The models that predict the group behaviour in the last phase of the games can help to identify groups that are going to provide socially sub-optimal contribution rates to the public good prior to their contributions. The ultimate goal could therefore be to intervene (e.g., nudges, efficiency providing formal institutions) only when the prediction based on a priori available information concludes that the group needs another push towards the social optimum. Given the specific structure of this financially incentivised laboratory experiment, the subjects act as free riders essentially only in the final stage of the experiment. The underlying dilemma of the public goods games is that socially and individually optimal behaviour do not coincide. The discrepancy becomes more apparent towards the end of the game when the benefits of cooperation decrease. In the last stage of the game there is no future cooperation, thus individuals in experiments without communication by majority maximise own payoffs by free-riding. Communication increases contribution rates on average, yet does not dispose the end-game effect completely $[3,5]$. We train a binary classifier that predicts whether the group will contribute fully in the very last period or defect. In this public goods experiment, each group had four participants; we assume that the end-game behaviour of each individual is influenced by the prior contributions of the other participants within his/her group. Therefore, we do not predict the contribution of each participant but of the entire group. The size of the dataset (described in Section 3) is small: it consists of 24 sessions including in total 127 different groups. The same subject might appear in several groups, but only in one session. For smaller datasets, deep networks tend to overfit. As a result, classical machine learning approaches often outperform deep networks for small datasets. That is why we use a classical approach here. To train person-independent models using classical machine learning approaches, we perform leave-one-session-out cross-validation. This ensures that the subjects do not appear in the training and test set simultaneously.

For the independently conducted content analysis, verbal conversations were first transcribed into texts. Subsequently, the texts were classified using binary content parameters, e.g., whether a specific topic [3] was raised (1) or not (0), and meta parameters of the conversations, e.g., individual and group word counts. The obtained variables were analysed with respect to the contribution behaviour and the collected demographic information.

The remainder of this paper is organised as follows. Section 1.1 describes the related works to address the relationship between this paper and the reference papers, while Section 1.2 describes the contribution of this work. Section 2 outlines the proposed automatic facial expression analysis approach. Section 3 describes the data and the experimental setup that was used to collect the data. Section 4 presents the experiments that were carried out, and their results. It also provides the results of the content analysis. We conclude the paper with a discussion in Section 5.

\subsection{Related Work}

Studies have shown that a large part of affective communication takes place either nonverbally or paralinguistically through audio or visual signals [6,7]. Besides the verbal information of opinions and desires, human interaction also involves nonverbal visual cues (through gestures, body posture, facial expressions and gaze) [8,9] which are widely and often unconsciously used in human communication $[10,11]$. Facial expressions are more than a static appearance cue with specific physical features. They are important signals of emotional states [12], communicate our intentions to others (as communication is often carried out face-to-face) [9,13-15], and aid in predicting human 
behaviour [16-18]. Facial expressions play an important role in interpersonal communication, and interpersonal behaviour of individuals is influenced by social context $[19,20]$. Interpersonal communication while displaying positive facial expressions increases individual performance in groups and contributes immensely to increasing, for example, workforce performance and overall organisational productivity [21]. In general, interpersonal communication has been proven to be very useful in different social groups. Facial expressions are used as an effective tool in behavioural research and widely used in cognitive science and social interactions.

Automatic analysis of social behaviour, in particular of face-to-face group conversations, is an emerging field of research in several domains such as human computer interaction, machine learning and computer vision $[16,22,23]$. The ultimate aim is to infer human behaviour by observing and automatically analysing the interaction of the group conversation taken from the audio and video channels. Jayagopi et al. (2009) describe a systematic study that investigates the characterisation of dominant behaviour in group conversations from fully automatic nonverbal (audio and visual) activity cues [24]. Jaques et al. (2016) present how a machine learning classifier can be trained using the facial expressions of one-minute segments of the conversation to predict whether a participant will experience bonding up to twenty minutes later [25]. Many automatic facial expressions were recognised on the basis of the display of individual facial Action Units (AUs) and their combinations. AUs are defined by the facial action coding system of Ekman and Friesen [26], which is the most commonly used method for coding facial expressions. Previous studies proved that combinations of AUs can account for more variation in behaviour than single AUs alone [27]. In addition, several lines of evidence suggest that the combination of AUs predict behaviour more precisely [28].

Research has shown that group behaviour in the first few minutes ( $3 \mathrm{~min}$ ) is highly correlated with the decisions made and actions taken in the future [29-31]. Thus, using machine learning coupled with computer vision allows computers to predict future behaviours. Recent research [32-35] shows how to automatically recognise emotions from videos or still images of individuals. However, group behaviour is barely addressed in the literature and studies do not deal with interpersonal communication and its impact on future decisions. Furthermore, at present, the typical publicly available facial expression databases contain recordings of spontaneous facial expressions and corresponding FACS annotations of one individual. But only one available database, the Sayette Group Formation Task (GFT) database, includes multiple interacting participants, despite the important role that facial expressions play in interpersonal communication. In GFT, there are three subjects in the videos interacting naturally and the purpose of this database is to estimate the overall emotion of the group interaction in order to study social interaction [36]. The originality of GFT is to have three subjects instead of two as in RECOLA [37]. However, with GFT, the future prediction of facial expressions for group participants is a difficult task, mainly because there are no direct decisions. Furthermore, there is no financial incentive involved in GFT to force decisions, so it cannot be utilised to study financial interactions. Ten Brinke et al. (2016) and Bonnefon et al. (2017) discuss the potential positive effects of incentivising experiments to detect deception or cooperation $[38,39]$. Therefore, in this paper, we utilise a special database from a laboratory public goods experiment of [40] that provides binary financial decisions after three minutes of FFC, as described in Section 2. Our prior research on the effect of communication on the voluntary contribution to public goods has clearly shown that communication for three minutes strongly increases the ability to cooperate, especially if the communication happens to be face-to-face [5]. This finding strongly supports the hypothesis that facial expressions play a major role in group communication processes.

Some researchers implement "human assessment" methods to predict the behaviour of subjects in laboratory experiments. In such cases, individuals attempt to guess whether other people will cooperate or not in setups similar to ours [41,42]. This paper is the first that investigates the facial expressions of a group in videos to predict their behaviour in a public goods experiment using facial expression recognition software. With respect to possible applications of public goods experiments, we refer to the prominent literature review by Chaudhuri [2]. In the broad sense, the results of such 
laboratory experiments are applied to, for example, charitable giving, managing natural resources, tax compliance and work relations. In a more narrow sense, the availability of tools automatically detecting free riding can aid (1) public transport systems that suffer losses due to fare evasion [43,44]; or (2) organisations that are interested in knowing whether a team will work together well, which has implications for group productivity [45].

\subsection{Contribution}

The main contribution of this paper is twofold. First, we introduce the application of computer vision and machine learning to experimental economics research in order to analyse group behaviour. Second, we introduce the economics topic of free riding in groups to the behaviour analysis community. Previous research has investigated the impact of communication on cooperation behaviour in groups [46]. Yet, so far, there has been no application that uses facial expressions to predict group behaviour after face-to-face communication (FFC) of free discussion. Thus, to the best of our knowledge, this is the first work that investigates the facial expressions of a group in videos to predict their behaviour in public goods experiment using facial expression recognition software. In this context, we propose an automatic facial expression analysis approach for the prediction of future group behaviour from pre-play FFC videos, which includes a novel group activity descriptor. Using the proposed approach, we show that a machine learning classifier can be trained to predict whether the group will fully contribute to the end of the games based on facial expression data from three minutes of communication. The spoken contents of the FFC video are investigated by categorising words in topics and using meta-data.

\section{Automatic Facial Expression Analysis Approach}

In the setup of a public goods game, all the group members benefit from the public good, regardless of their own contribution [4]. Within the scope of this study, the participants communicate with each other prior to secretly choosing how much of their financial endowment they want to put into the public pot. Both facial expressions and content analysis are conducted independently to investigate whether the FFC induces the group members to sustain cooperation and stop free riding. Figure 1 shows an overview of the automatic facial expression analysis for the FFC video to predict the end-game behaviour of the groups. Each group in the FFC video has four participants (subjects). We do not predict the contribution of each subject but of the entire group. First, we use OpenFace [47] to extract head pose and AUs features (facial features, FF) from each frame for each participant (see Section 2.1). Second, the FF are condensed into descriptors [48] for each individual face from all the frames (see Section 2.2). Third, the descriptors for each of the 4 individuals are concatenated in all possible ways to form the group activity descriptors and all of them receive the same label (see Section 2.3). Finally, we classify the group activity descriptors individually and the outcomes are evaluated and averaged to calculate the classification performance (see Section 2.4). 


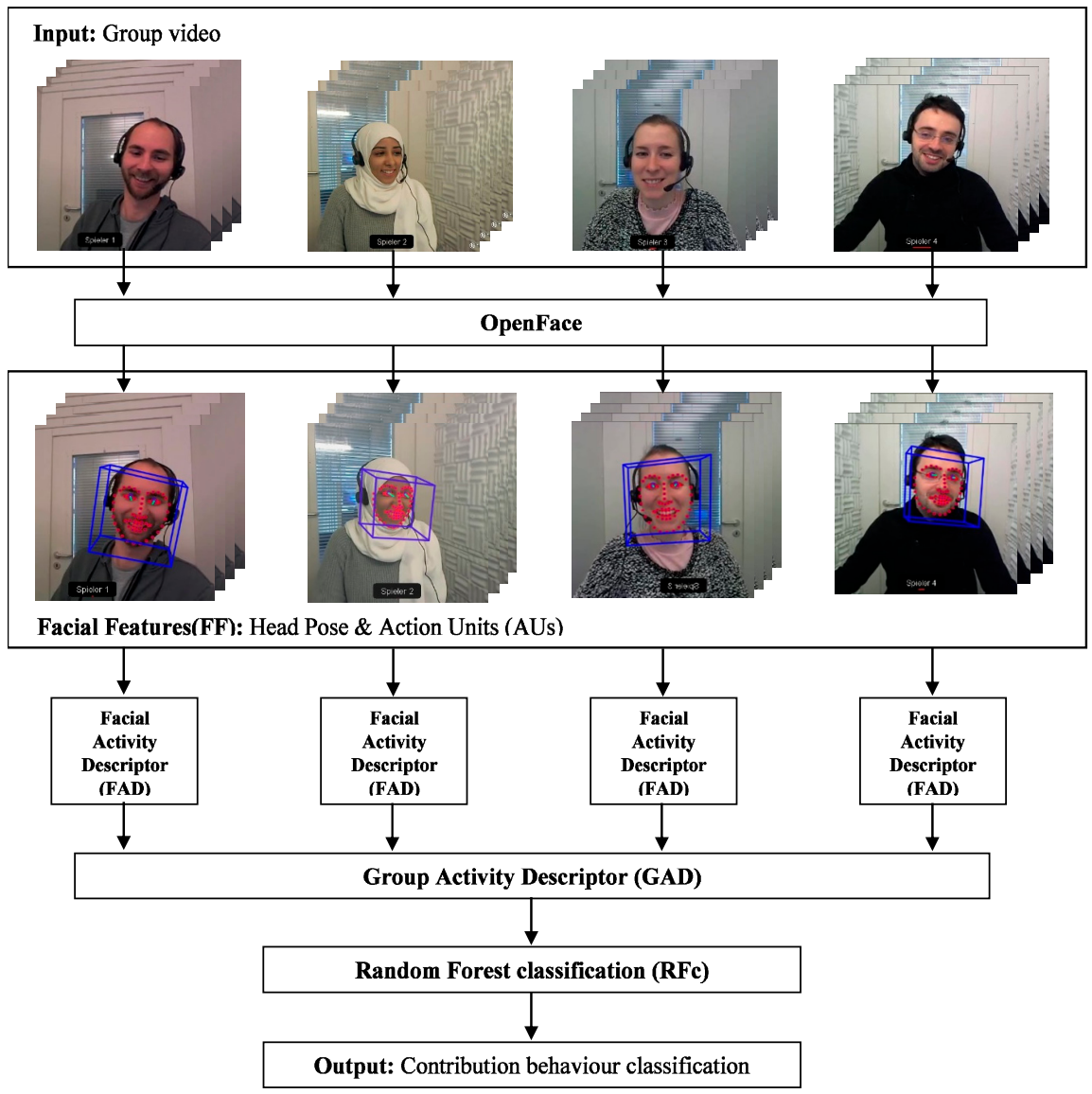

Figure 1. The pipeline of automated facial expression analysis from FFC videos. Each input group video consists of 4 participants communicating for three minutes (in total we have 127 different groups). Each face is first analysed using OpenFace. OpenFace detects the face, facial landmarks, head pose, and extracts facial action units (AUs). AUs and head pose form the facial features for each participant. From these, also for each participant, we calculate facial activity descriptors (FADs). All FADs from all 4 participants are then combined to form the group activity descriptor (GAD). Finally, random forest $(\mathrm{RF})$ is used to classify the facial contribution behaviour (predicting whether the group will contribute fully or not).

\subsection{Facial Features (FF)}

The first step in our analysis pipeline uses OpenFace [47] to extract the facial features from each individual face and frame. OpenFace detects the face, facial landmarks, extracts Action Units (AUs), and estimates head pose (see Figure 1). The frame-based AUs and head pose form the facial features. To analyse the importance of the features we group them into four subsets as follows. FF1: the first category includes all features (head position, AU presence, and AU intensity); FF2: includes head pose and AU presence features (HP \& AUP); FF3: includes head poses and AU intensity features (HP \& AUI); FF4: includes manually selected features that OpenFace can estimate robustly based on the study of [47] (see Table 1). 
Table 1. List of extracted facial features in OpenFace (I-intensity, $\mathrm{P}$ - presence).

\begin{tabular}{|c|c|c|c|c|c|c|c|c|}
\hline \multirow{2}{*}{$\begin{array}{c}\text { Head Pose } \\
\text { Yaw }\end{array}$} & \multirow{2}{*}{$\begin{array}{c}\text { AU } \\
\text { AU1 }\end{array}$} & \multirow{2}{*}{$\begin{array}{c}\text { AU Full Name } \\
\begin{array}{c}\text { Inner brow } \\
\text { raiser }\end{array}\end{array}$} & \multicolumn{2}{|c|}{ Prediction } & \multirow{2}{*}{$\begin{array}{c}\text { AU } \\
\text { AU14 }\end{array}$} & \multirow{2}{*}{$\begin{array}{c}\text { AU Full Name } \\
\text { Dimpler }\end{array}$} & \multicolumn{2}{|c|}{ Prediction } \\
\hline & & & I & $\mathrm{P}^{*}$ & & & $\mathrm{I}^{*}$ & $\mathrm{P}$ \\
\hline Pitch & AU2 & $\begin{array}{l}\text { Outer brow } \\
\text { raiser }\end{array}$ & $\mathrm{I}^{*}$ & $\mathrm{P}^{*}$ & AU15 & $\begin{array}{l}\text { Lip corner } \\
\text { depressor }\end{array}$ & $\mathrm{I}^{*}$ & $\mathrm{P}$ \\
\hline \multirow[t]{7}{*}{ Roll } & AU4 & Brow lowerer & I & $\mathrm{P}^{*}$ & AU17 & Chin raiser & $\mathrm{I}^{*}$ & $\mathrm{P}$ \\
\hline & AU5 & $\begin{array}{l}\text { Upper lid } \\
\text { raiser }\end{array}$ & I & $\mathrm{P}^{*}$ & AU20 & Lip stretched & $I^{*}$ & $\mathrm{P}^{*}$ \\
\hline & AU6 & Cheek raiser & I & $\mathrm{P}$ & AU23 & Lip tightener & $\mathrm{I}^{*}$ & $\mathrm{P}^{*}$ \\
\hline & AU7 & Lid tightener & $\mathrm{I}^{*}$ & $\mathrm{P}$ & AU26 & Jaw drop & $\mathrm{I}$ & $\mathrm{P}^{*}$ \\
\hline & AU9 & Nose wrinkler & I & $\mathrm{P}^{*}$ & AU28 & Lip suck & - & $\mathrm{P}^{*}$ \\
\hline & AU10 & $\begin{array}{l}\text { Upper lip } \\
\text { raiser }\end{array}$ & I & $\mathrm{P}$ & AU45 & Blink & $\mathrm{I}^{*}$ & $\mathrm{P}^{*}$ \\
\hline & AU12 & $\begin{array}{l}\text { Lip corner } \\
\text { puller }\end{array}$ & I & $\mathrm{P}$ & & & & \\
\hline
\end{tabular}

${ }^{*}$ Refers to the selected facial features that OpenFace can estimate robustly based on the results of [47].

\subsection{Facial Activity Descriptors (FADs)}

For each participant and video, a facial activity descriptor is extracted from the corresponding facial features, as described in Section 2.2 of [49]. In brief, the FF (which differ in length) are condensed in descriptors [48]: First, we smooth each FF with a Butterworth filter (first order, cutoff $1 \mathrm{~Hz}$ ); Second, we calculate the first and second derivative of the smoothed signal [49]; Third, we extract 16 statistics from the smoothed signals and both derivatives for each facial feature (among others: mean, maximum, standard deviation, time of maximum value and duration in which the time series values are above their mean). In most experiments, we calculate the FAD for each individual from all the frames of the FFC video. However, to analyse the usefulness of specific video parts for the classification, we also extract FADs from temporal segments (as detailed in Section 4).

\subsection{Group Activity Descriptors (GADs)}

The GAD is used to predict group behaviour of the FFC. After we calculate the four FADs of each group, we concatenate them in all $4 !=24$ permutations (e.g., 1234, 1243, 1432, . . ) to form the GADs. This way, each group conversation is represented by 24 instances and each is given the group label. This obviously increases the dataset size, which is favourable because the dataset with 127 FFC videos is quite small. The feature space dimensionality of each GAD for FF1 is 7,296, for FF2 is 4,032, for FF3 is 3,840 and for FF4 is 4,032 .

\subsection{Classification}

The $(127 \times 24=3048)$ GADs are classified individually. The classifier uses leave-one-session-out (24-session) cross-validation, i.e., a subject never appears in a training and test set simultaneously. Cross-validation is a very useful technique for assessing the effectiveness of our models to mitigate overfitting. We hold out the GADs of one session for testing while the rest form the training set. We train the model on the training set and evaluate it on the test set. The cross-validation process is then repeated 24 times, with each of the sessions used exactly once as the test set. In total, 24 sessions provide 24 training sets and 24 test sets. The results of test sets are averaged to form the final performance. 


\section{Dataset}

This section introduces the parts of a larger experiment that are relevant to this paper. The collected data and the experimental setup are detailed in $[5,40]$. The experimental design was executed in z-Tree [50]. The experiment was organised and recruited with the software hroot [51] in the Magdeburg Experimental Laboratory of Economic Research (MaXLab). The participants were students from the Otto-von-Guericke University Magdeburg. The entire experiment consisted of three blocks. The second and third blocks, where the participants had the chance to communicate face-to-face via audio-video communication software prior to the voluntary contribution mechanism (VCM), were analysed. During the communication period of three minutes, the participants were free to discuss anything. The dataset consisted of 127 different groups divided into 24 sessions, with each group having 4 participants (subjects). It was possible for the same subject to appear in several groups, but only in the same session. For the evaluation, we suggest leave-one-session-out cross validation, which avoids subject overlap between the training and the test set and approximates generalisation performance with unseen subjects. For this purpose, the 24 sessions of our dataset were divided into two subsets (training and test set) 24 times: each time a different session formed the test set and the rest formed the training set. Behaviour labels (whether the group contributes fully or not) were provided for both training and test sets, while training set labels were used to learn the classification model and test set labels are used to measure the performance of a classification.

\section{Experiments and Results}

This section provides the experiments and results of the group's contributions in the public goods games after a group conversation. Section 4.1 describes the setup of experiments and the results. Ablation experiments are described in Section 4.2. Section 4.3 shows the feature importance of FF4, and finally, Section 4.4 discusses the content analysis experiment.

\subsection{Experimental Setup and Results}

We conducted several experiments in order to gain insights into the contribution behaviour classification and to improve the classification accuracy. For reference, we calculate a trivial classier which always votes for the majority class of the training set. Note that this is also done within the leave-on-session-out cross-validation, providing 24 different results (depending on the class distributions of the test sets). This informed guess allows us to have a meaningful comparison with our models.

\subsubsection{Model and Feature Selection}

The results in Figure 2a show that RFc performs much better than Support Vector Machine (SVM) models. This is consistent to the results of [52] in which classification rates with RFc were significantly better than with SVM. The SVM models perform worse than trivial. This suggests that a large number of features contain noise, which significantly harms the SVM performance (while RFc is quite robust against noise). This also seems to hold true for the different sets of facial features. The selected features (FF4) probably contain less noise than others and thus perform best using the SVM. However, RFc performs better using all features (FF1) and using the selected feature subset (FF4). Therefore, we continue using RFc with FF1 and FF4. 


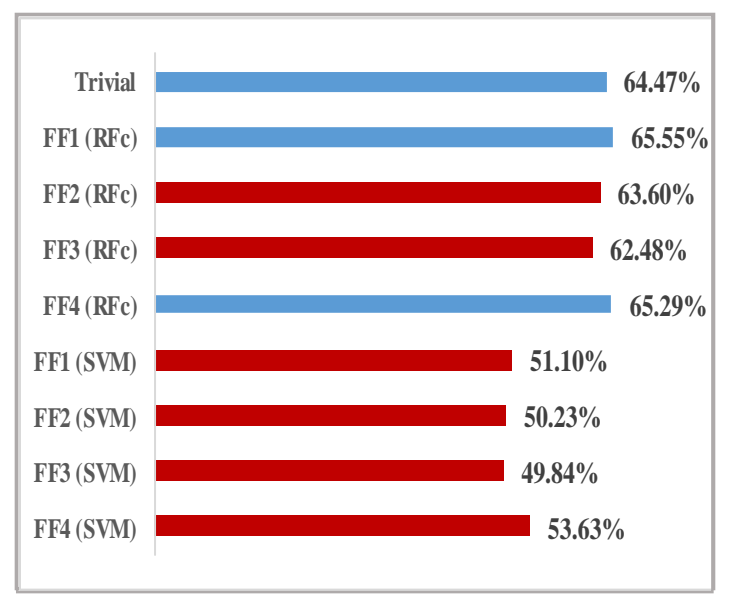

(a)

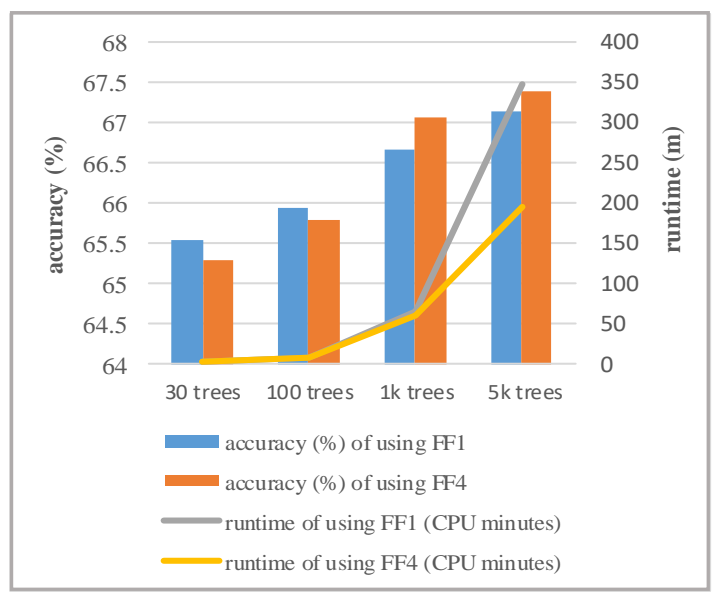

(b)

Figure 2. The accuracy of RFc and SVM: (a) The accuracy of RFc (30 trees), SVM, and informed guess (trivial) using leave-one-session-out crossvalidation; (b) The accuracy and runtime of RFc using FF1 and FF4 with varying number of trees.

\subsubsection{RFc Trees}

We investigate the influence of the number of trees on performance and training time. We train 100, 1k, and 5k trees both on FF1 and FF4. The results in Figure 2b show that the accuracy for FF1 and FF4 increases with the number of trees. However, the training time also increases. The runtime in minutes for the entire 24-session cross-validation procedure of training and test sets was plotted to show the factor of runtime increase when the parameters increase. Since the performances gain plateaus at $5 \mathrm{k}$ trees, we stopped using more. Models that use FF4 with $1 \mathrm{k}$ and $5 \mathrm{k}$ trees are better than the models that use FF1 because RFc is more effective when using a small subset of high-dimensional features. Therefore, we continue using RFc (5k trees) with FF4.

\subsubsection{Optimal Threshold}

In the previous experiments, the RFc is trained on all the GADs of the training set. Each group conversation is represented by $4 !=24$ samples and each is given the same label (see Section 2.3). However, the RFc is not aware of this fact and might predict samples of the same group differently. We average the binary prediction outcome of all (4!) samples of the same group and threshold this average value to get the prediction result for that particular group. We determine an optimal threshold that maximises accuracy on the training set.

We calculate the optimal threshold and estimate the final performance by using nested cross-validation. The hyper-parameter search is performed with inner cross-validation, while outer cross-validation computes an unbiased estimate of the expected accuracy of the RFc with the optimised threshold. For each fold of the outer cross validation, the inner cross-validation runs on the outer cross-validation's training set, trains 23 RFc models and tests them with a predefined set of thresholds. The threshold that yields best accuracy (mean across the 23 inner test sets) is selected as the optimal threshold. In outer cross-validation, we apply RFc with the optimal threshold to the remaining session $(1 / 24)$ of the data (test set) and calculate the accuracy. In total, we get 24 results, which are averaged to form the final performance. This double cross-validation procedure provides an unbiased estimate of the accuracy for the RFc.

Applying the optimal threshold increases the accuracy of the RFc (5k trees) model (using FF4) from $67.39 \%$ to $68.11 \%$. Therefore, we continue using RFc (5k trees) with FF4 and optimal threshold. 


\subsubsection{Temporal Splits}

Computing the FADs for the entire video (see Section 2.2) might reduce the possibility of obtaining time-related features. Because the FADs primarily contain statistical features, it is possible that changes in the signals over time are not captured well enough. Instead of computing the FADs for the entire video, we divide each FFC video into 3, 4 and 5 equally long videos (splits). We extract the FADs and GADs from FF4 of these 12 different splits $(3+4+5=12)$ and combine the GADs in multiple combinations. We train a model for each split and multiple combinations (33 in total, Table 2 shows the full list of splits). In this way, we can capture more time-related features from FFC. We introduce three different categories containing models from different split combinations (beginning models, end models and a combination of beginning and end models). This way we can investigate if there are some parts of the FFC video that are more useful than others.

Table 2. The three categories of splits (combined models, beginning and end models) to investigate which categories are most useful. Each split is processed individually by RFc (5k trees) using FF4 and optimal threshold.

\begin{tabular}{cccccc}
\hline \multicolumn{2}{c}{ Combined Models (11 Splits) } & Beginning Models (11 Splits) & \multicolumn{2}{c}{ End Models (11 Splits) } \\
\hline $\begin{array}{c}\text { 1st \& 3rd third } \\
\text { split }\end{array}$ & $\begin{array}{c}\text { 1st \& 4th \& 5th } \\
\text { fifth split }\end{array}$ & 1st third split & 3rd fifth split & 3rd third split & 5th fifth split \\
\hline $\begin{array}{c}\text { 1st \& 4th } \\
\text { quarter split }\end{array}$ & $\begin{array}{c}\text { 1st \& 2nd \& 4th } \\
\text { \& 5th fifth split }\end{array}$ & 1st quarter split & $\begin{array}{c}\text { 1st \& 2nd fifth } \\
\text { split }\end{array}$ & $\begin{array}{c}\text { 3rd quarter } \\
\text { split }\end{array}$ & $\begin{array}{c}\text { 3rd \& 4th fifth } \\
\text { split }\end{array}$ \\
\hline $\begin{array}{c}\text { 1st \& 3rd \& 4th } \\
\text { quarter split }\end{array}$ & $\begin{array}{c}\text { 1st \& 2nd \& 3rd } \\
\text { \& 5th fifth split }\end{array}$ & $\begin{array}{c}\text { 2nd quarter } \\
\text { split }\end{array}$ & $\begin{array}{c}\text { 1st \& 3rd fifth } \\
\text { split }\end{array}$ & $\begin{array}{c}\text { 4th quarter } \\
\text { split }\end{array}$ & $\begin{array}{c}\text { 3rd \& 5th fifth } \\
\text { split }\end{array}$ \\
\hline $\begin{array}{c}\text { 1st \& 2nd \& 4th } \\
\text { quarter split }\end{array}$ & $\begin{array}{c}\text { 1st \& 3rd \& 4th } \\
\text { \& 5th fifth split }\end{array}$ & $\begin{array}{c}\text { 1st \& 2nd } \\
\text { quarter split }\end{array}$ & $\begin{array}{c}\text { 2nd \& 3rd fifth } \\
\text { split }\end{array}$ & $\begin{array}{c}\text { 3rd \& 4th } \\
\text { quarter split }\end{array}$ & $\begin{array}{c}\text { 4th \& 5th fifth } \\
\text { split }\end{array}$ \\
\hline $\begin{array}{c}\text { 1st \& 5th fifth } \\
\text { split }\end{array}$ & $\begin{array}{c}\text { 1st \& 2nd \& 3rd } \\
\text { \& 4th \& 5th } \\
\text { fifth split }\end{array}$ & 1st fifth split & $\begin{array}{c}\text { 1st \& 2nd \& 3rd } \\
\text { fifth split }\end{array}$ & 3rd fifth split & $\begin{array}{c}\text { 3rd \& 4th \& 5th } \\
\text { fifth split }\end{array}$ \\
\hline $\begin{array}{c}\text { 1st \& 2nd \& 5th } \\
\text { fifth split }\end{array}$ & & 2nd fifth split & & 4th fifth split & \\
\hline
\end{tabular}

Figure 3 shows the comparison of accuracy between the uninformed guess $(50 \%)$, the informed guess (trivial) and three categories of models (combined, beginning and end models). These models are trained using RFc (5k trees) with FF4 and use optimal threshold. The end models perform best in terms of classification performance compared to the uninformed guess, trivial, combined, and beginning models. The end models probably contain more informative features than others. On average, end models predict about $70.17 \%$ of the decisions correctly, which is significantly more than guessing, while the combined models predict about $68.57 \%$, the beginning models predict about $68.40 \%$ and the trivial models predict about $64.47 \%$. Table 3 shows the results of end models are the best based on p-values, which were obtained through a paired U-test. The results are significant compared with the uniformed guess and trivial models. Other differences failed to reach the significance level, but overall the results are promising for further research. 


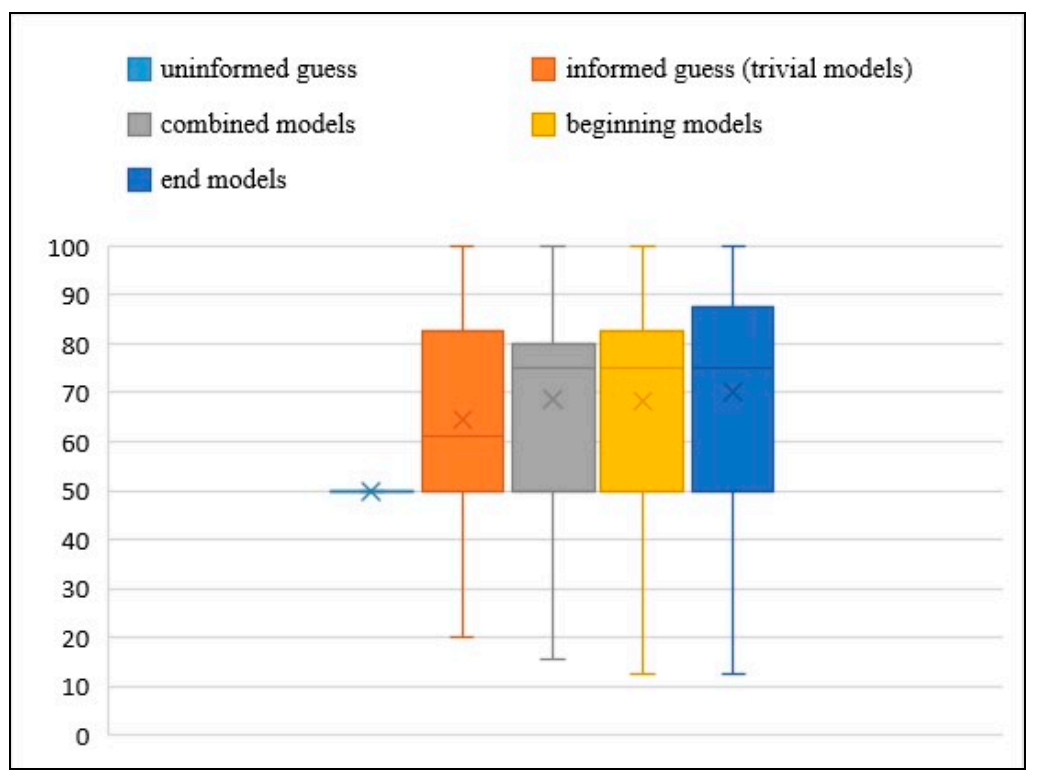

Figure 3. Boxplot of comparing uninformed guess and the accuracy of trivial models with three different RFc (5k trees) models that use FF4 and optimal threshold (combined, beginning and end models). The accuracy of all the RFc models is better than the uninformed guess and the trivial models, with end models obtaining the best accuracy, followed by combined models and beginning models. The crosses represent mean values, the boxes show $25 \%$ and $75 \%$ quantiles and the median, while the whiskers show the minimum and maximum values.

Table 3. Paired U-test for comparing the results of the uniformed guess, trivial, combined, beginning and end models using RFc (5k) with FF4 and optimal threshold.

\begin{tabular}{cc}
\hline Models & p-Value \\
\hline uninformed guess \& trivial models & $0.0000^{*}$ \\
uninformed guess \& combined models & $0.0000^{*}$ \\
uninformed guess \& beginning models & $0.0000^{*}$ \\
uninformed guess \& end models & $0.0000^{*}$ \\
trivial models \& combined models & 0.0530 \\
trivial models \& beginning models & 0.0519 \\
trivial models \& end models & $0.0082^{*}$ \\
combined models \& beginning models & 0.9872 \\
combined models \& end models & 0.4435 \\
beginning models \& end models & 0.5147 \\
\hline \multicolumn{2}{c}{$\mathrm{p}<0.05$ (significant difference). }
\end{tabular}

\subsection{Ablation Studies}

To gain further insights into the improvements obtained by the proposed approach, we summarise the experiments for ablation studies as listed in Table 4, where we aim to examine the effectiveness and contributions of different strategies used in the proposed approach. 
Table 4. An overview of the ablation studies of the proposed solutions, with each experiment using RFC with FF4. We conducted 5 experiments combining different methods (ticks display its usage) to improve the model's performance. The results are the average accuracy of models obtained from cross-validation.

\begin{tabular}{cccccc}
\hline Method & Exp. 1 & Exp. 2 & Exp. 3 & Exp. 4 & Exp. 5 \\
\hline Using all frames with RFc & $\sqrt{ }$ & $\sqrt{ }$ & $\sqrt{ }$ & & \\
Using RFc with 5k trees & & $\sqrt{ }$ & $\sqrt{ }$ & $\sqrt{ }$ & $\sqrt{ }$ \\
Applying optimal threshold & & & $\sqrt{ }$ & $\sqrt{ }$ & $\sqrt{ }$ \\
Using combined models & & & & $\sqrt{ }$ & $\sqrt{ }$ \\
Using end models (11 splits) & & & & & $68.57 \%$ \\
\hline Average Accuracy & $65.29 \%$ & $67.39 \%$ & $68.11 \%$ & $70.18 \%$ \\
\hline
\end{tabular}

Table 4 provides an overview of a series of improvements in RFc models that use the FF4 after applying a series of experiments. The average accuracy is increased from about $65 \%$ to $70 \%$. In experiment 1 , in which we use all the frames in the FFC video to train RFc (30 trees) with FF4, the models correctly predict $65.29 \%$ of decisions, which is slightly better than the informed guess $(64.47 \%)$. In experiment 2 , we use the same setting of experiment 1 except that we increase the number of RFc trees to $5 \mathrm{k}$ to increase accuracy. Additionally, the optimal threshold is applied in experiment 3 and prediction accuracy is increased to about $68.11 \%$. In experiment 4 , we train RFc (5k trees) using FF4, which are extracted from different splits and combinations in the FFC video to capture the differences between different splits (by obtaining more time-related features) with the optimal threshold. We train RFc (5k trees) with 11 different splits of the combined models (see Table 2). The average accuracy of combined models is $68.57 \%$, while the accuracy of the all frames model is about $68.11 \%$. These results indicate that some splits of the FFC video are not useful for classification. In experiment 5 , we investigate whether the first or the last part of the FFC video is most informative. We train RFc (5k trees) with 22 different splits (see Table 2) belonging to the first and the last parts of the FFC video. The beginning models predict about $68 \%$, while the end models predict about $70 \%$, which is significantly more than guessing. However, the accuracies of the end models are not significantly higher than the accuracies of the beginning models.

\subsection{Feature Importance in RFC}

Table 5 shows the feature importance values of FF4 calculated by using RFc (5k), with the values sorted in descending order. Head pose features turned out to be the most important features, followed by most of the AU intensity features (AU45 = blinking, AU20 = horizontal lip stretching, AU23 = tightening of lips, AU17 = chin raising, AU02 = outer brow raising, and AU15 = lip corner depressing), and finally the AU presence features. The results indicate that head pose and intensity features are more informative for behavioural prediction than the presence features are. We believe that the head pose might be particularly informative because it can show the subjects lack of engagement in the conversation. Nevertheless, more data would be necessary for a detailed investigation.

Table 5. Features importance for the FF4 by using RFc (5k), I - intensity, P - presence. The features in table are ordered by their importance from most important to least important.

\begin{tabular}{cccccccccccc}
\hline Imp & Feature & Value & Imp & Feature & Value & Imp & Feature & Value & Imp & Feature & Value \\
\hline 1 & pose_y & 0.1387 & 7 & AU17_I & 0.1029 & 13 & AU07_I & 0.0759 & 19 & AU26_P & 0.0696 \\
2 & pose_p & 0.1367 & 8 & AU02_I & 0.1022 & 14 & AU20_P & 0.0744 & 20 & AU09_P & 0.0667 \\
3 & pose_r & 0.1349 & 9 & AU15_I & 0.1012 & 15 & AU45_P & 0.0736 & 21 & AU01_P & 0.0640 \\
4 & AU45_I & 0.1060 & 10 & AU23_P & 0.0769 & 16 & AU02_P & 0.0721 & & & \\
5 & AU20_I & 0.1036 & 11 & AU05_P & 0.0766 & 17 & AU28_P & 0.0717 & & & \\
6 & AU23_I & 0.1033 & 12 & AU14_I & 0.0763 & 18 & AU04_P & 0.0707 & & & \\
\hline
\end{tabular}




\subsection{Content Analysis}

We analysed the content of the verbal conversation by first transcribing the spoken language into texts and subsequently classifying the texts using binary content parameters, e.g., whether a specific topic [3] was raised (1) or not (0), and meta parameters of the conversations, e.g., individual and group word counts. The obtained variables were analysed with respect to the contribution behaviour and the collected demographic information using a Tobit regression. Table 6 illustrates that the variable "End-Games" has the strongest effect on the group level contributions. As is described in greater detail and discussed with respect to other parameters in [40], the variable codes whether the individuals mentioned the end games specifically. Briefly discussing the idea of making high contributions until the very end has a significant, positive effect on the contributions. This result is, furthermore, possibly in line with the obtained observation that the last parts of FFC are more informative. The conclusion that providing high contributions to the public good until the very end constitutes the final element of the arrangement in the group (for example, 1 . What is the optimal contribution level? 2. Does everyone agree on the discussed scheme? 3. Identify the special trait of the last period). This is further supported by the observation that groups that discussed longer in terms of the word counts were on average more successful. However, this effect is stronger when the groups communicate for the first time and weaker when they repeat the communication in the next round. This may be a result of a learning process [40].

Table 6. Results of Tobit regression of group contributions with p-values denoted in brackets.

\begin{tabular}{cccc}
\hline $\begin{array}{c}\text { Dependent Variable: } \\
\text { Group Contributions }\end{array}$ & $\begin{array}{c}\text { Coefficients of Joint } \\
\text { Observations }\end{array}$ & $\begin{array}{c}\text { Dependent Variable: } \\
\text { Group Contributions }\end{array}$ & $\begin{array}{c}\text { Coefficients of Joint } \\
\text { Observations }\end{array}$ \\
\hline Number of words & $0.088(0.102)$ & Number of males & $-4.973(0.361)$ \\
End-Games & $39.855(0.006)$ & Aggregated age & $0.108(0.887)$ \\
Invest All & $64.899(0.073)$ & Constant & $3.395(0.964)$ \\
Subjects Against & $-23.850(0.205)$ & R-square & 0.0447 \\
Previous Experience & $6.795(0.554)$ & Number of Observations & 127 \\
Threats \& Consequences & $-7.159(0.604)$ & LR-Chi2 & 23.01 \\
Number of economists & $1.349(0.819)$ & & \\
\hline
\end{tabular}

\section{Discussion}

In this paper, we proposed two approaches (a facial expression analysis approach and a content analysis approach) to investigate what influences subjects' contributions in a public goods game utilising a dataset of 127 groups, each group with 4 participants. We automatically analyse the facial expressions of the FFC videos in three steps. First, we extract facial features from each individual face and frame by using OpenFace [47] (see Section 2.1). Second, we calculate the facial activity descriptors (FADs) for each individual face from all the frames (see Section 2.2). Third, we concatenate the activity descriptors of the four individuals in all $4 !=24$ possible ways to form our newly proposed group activity descriptors (GADs) (see Section 2.3). All the GADs receive the same label. We classify all 127 videos $\times 24$ permutations $=3048$ GADs individually and average the classification outcome of each video to obtain the prediction score (see Section 2.4).

Several experiments in facial expression analysis of FFC were conducted, trying to predict the full-contribution behaviour better than uninformed and informed guessing. The results show that predicting the contribution behaviour of the group based on the facial visual cues communication is challenging; the results were better than uniformed guesses and slightly better than informed guesses (trivial). We expected this task to be particularly difficult because we are trying to predict decisions that have far more hidden influences. The initial results presented are promising and we believe that a larger dataset is necessary to predict cooperation behaviour more reliably. This hypothesis is supported by the results in Figure $2 \mathrm{~b}$. The accuracies of high capacity classifiers (RFc with $1 \mathrm{k}$ and $5 \mathrm{k}$ trees) improve when only a subset of the features (FF4) is used, i.e., the prediction becomes more reliable although less information is available. This behaviour is related to the curse of dimensionality and Hughes phenomenon [53] and is typical for datasets that are too small. Increasing dataset size 
would offer great potential for more reliable classification in higher dimensional feature spaces and for exploring fine-grained behavioural patterns that help sustain cooperation and avoid free riding in public goods games. In turn, this would find application in public tasks, e.g., policies for the solution of environmental problems or managerial analyses concerning the performance of teams in companies.

We initially assumed that the beginning of the FFC video was more informative when it came to predicting human behaviour, perhaps even just the first seconds. Our findings did not support this assumption, with both the facial expression analysis and content analysis approaches indicating that the last part of the FFC video was probably more informative. However, we were not able to prove this, probably due to an issue of the dataset size.

We looked at the correctly classified and wrongly classified FFC video and found little difference in the behaviour between groups at the beginning of the FFC video. However, groups that do not contribute fully show less commitment later in the FFC video. Therefore, we conclude that the last part of the FFC video can be used to predict the contribution behaviour of groups more reliably because it is easier to establish whether the group is communicating well when the introductory phase has already ended. Furthermore, participants might control their facial expressions more at the beginning of communication, while their spontaneous facial expressions become visible at the end of the communication. Thus, facial expressions and head movements provide valuable information that contributes to the classification of behaviour. In short, the current results show how it is possible to predict whether all the participants in a group will contribute fully in the last period of the public goods game. Although facial expression analysis and content analysis show that the final part of FFC videos seems to be more advantageous for predicting the contribution behaviour of groups, we have no proof that the last part of FFC videos are better than the beginning of the FFC videos due to a lack of data. It is essential to use larger datasets to obtain a deeper understanding of the problem. This would allow cooperation behaviour to be predicted more reliably and our findings to be confirmed.

Author Contributions: E.O. designed and performed the execution of automatic facial expression analysis; D.B. designed the experimental setup with face-to-face communication and performed the execution of content analysis experiment; original draft preparation: E.O., D.B., F.S.; conceptualisation and methodology: F.S., P.W., A.A.-H. and J.W.; writing, review and editing: all authors.

Funding: This research is supported by German Academic Exchange Service (DAAD), the Federal Ministry of Education and Research (BMBF, projects 03ZZ0443G, 03ZZ0471A and 03ZZ0470), and the innovations fund of the OvGU. The experiment was funded by the IWH-Explore-Projekt SP2015-03. The sole responsibility for the content lies with the authors.

Conflicts of Interest: The authors declare no conflict of interest.

\section{References}

1. Balliet, D. Communication and Cooperation in Social Dilemmas: A Meta-Analytic Review. J. Confl. Resolut. 2010, 54, 39-57. [CrossRef]

2. Chaudhuri, A. Sustaining cooperation in laboratory public goods experiments: A selective survey of the literature. Exp. Econ. 2011, 14, 47-83. [CrossRef]

3. Brosig, J.; Weimann, J.; Ockenfels, A. The effect of communication media on cooperation. Ger. Econ. Rev. 2003, 4, 217-241. [CrossRef]

4. Public Goods Game. Available online: https://en.wikipedia.org/w/index.php?title=Public_goods_game\& oldid=892553907 (accessed on 18 June 2019).

5. Altemeyer-Bartscher, M.; Bershadskyy, D.; Schreck, P.; Timme, F. Endogenous Institution Formation in Public Good Games: The Effect of Economic Education. Available online: https://hdl.handle.net/10419/173200 (accessed on 18 June 2019).

6. Mehrabian, A. Silent Messages; Wadsworth: Belmont, CA, USA, 1971; pp. 40-56, ISBN 0-534-00059-2.

7. Mehrabian, A.; Ferris, S.R. Inference of attitudes from nonverbal communication in two channels. J. Consult. Psychol. 1967, 31, 248-252. [CrossRef] [PubMed]

8. Knapp, M.L.; Hall, J.A. Nonverbal Communication in Human Interaction; Wadsworth Publishing: Belmont, CA, USA, 2009; pp. 59-88, ISBN 9780495568698. 
9. Zhang, S.; Zhao, X.; Lei, B. Robust Facial Expression Recognition via Compressive Sensing. Sensors 2012, 12, 3747-3761. [CrossRef]

10. Aran, O.; Hung, H.; Gatica-Perez, D. A Multimodal Corpus for Studying Dominance in Small Group Conversations. In Proceedings of the LREC workshop on Multimodal Corpora: Advances in Capturing, Coding and Analyzing Multimodality, Valletta, Malta, 18 May 2010; pp. 2-6.

11. Horgan, T.G.; Hall, J.A.; Knapp, M.L. Non-Verbal Communication in Human Interaction, 8th ed.; Wadsworth: Belmont, CA, USA; Cengage Learning: Boston, MA, USA, 2014; Volume 8, p. 528, ISBN 9781133311591.

12. Ekman, P. Emotion in the Human Face, 2nd ed.; Cambridge University Press: Cambridge, UK, 1982; pp. $178-211$.

13. Keltner, D.; Haidt, J. Social functions of emotions at four levels of analysis. Cogn. Emot. 1999, 13, 505-521. [CrossRef]

14. Pantic, M. Machine Analysis of Facial Behaviour: Naturalistic \& Dynamic Behaviour 2. The Process of Automatic Facial Behaviour Analysis. Philos. Trans. R. Soc. Lond. Ser. B Biol. Sci. 2009, 364, 3505-3513. [CrossRef]

15. Paul, E. Facial expression and emotion. Am. Psychol. 1993, 48, 384-392.

16. Aran, O.; Gatica-Perez, D. Analysis of Group Conversations: Modeling Social Verticality. In Computer Analysis of Human Behaviored; Salah, A.A., Gevers, T., Eds.; Springer: Berlin/Heidelberg, Germany, 2011; pp. 293-323, ISBN 978-0-85729-993-2.

17. Krumhuber, E.; Manstead, A.S.R.; Cosker, D.; Marshall, D.; Rosin, P.L.; Kappas, A. Facial Dynamics as Indicators of Trustworthiness and Cooperative Behavior. Emotion 2007, 7, 730-735. [CrossRef]

18. Nalini, A.; Robert, R. Thin slices of expressive behavior as predictors of interpersonal consequences: A meta-analysis. Psychol. Bull. 1992, 111, 256-274.

19. Forgas, J.P.; Jones, R. Interpersonal Behaviour: The Psychology of Social Interaction; Pergamon Press: Elmsford, NY, USA, 1985; ISBN 9780080298542.

20. Jacques, J.C.S.; Güçlütürk, Y.; Marc, P.; Güçlü, U.; Andújar, C.; Baró, X.; Escalante, H.J.; Guyon, I.; Gerven, M.V.; Lier, R.V.; et al. First Impressions: A Survey on Computer Vision-Based Apparent Personality Trait Analysis. arXiv 2018, arXiv:1804.08046.

21. Okoro, E.; CWashington, M.; Thomas, O. The Impact of Interpersonal Communication Skills on Organizational Effectiveness and Social Self-Efficacy: A Synthesis. Int. J. Lang. Linguist. 2017, 4, 28-32.

22. Gatica-Perez, D. Automatic nonverbal analysis of social interaction in small groups: A review. Image Vis. Comput. 2009, 27, 1775-1787. [CrossRef]

23. George, S.; Pascal, L. An approach to automatic analysis of learners' social behavior during computer-mediated synchronous conversations. In Proceedings of the International Conference on Intelligent Tutoring Systems, Biarritz, France, 2-7 June 2002; pp. 630-640.

24. Jayagopi, D.B.; Hung, H.; Yeo, C.; Gatica-Perez, D. Modeling Dominance in Group Conversations Using Nonverbal Activity Cues. IEEE Trans. Audiospeechand Lang. Process. 2009, 17, 501-513. [CrossRef]

25. Jaques, N.; McDuff, D.; Kim, Y.L.; Picard, R. Understanding and predicting bonding in conversations using thin slices of facial expressions and body language. In Proceedings of the International Conference on Intelligent Virtual Agents, Los Angeles, CA, USA, 20-23 September 2016; pp. 64-74.

26. Ekman, P.; Friesen, W.V. Facial Action Coding System: A Technique for the Measurement of Facial Movement; Consulting Psychologists Press: Palo Alto, CA, USA, 1978; Volume 1.

27. Bartlett, M.; Littlewort, G.; Vural, E.; Lee, K.; Cetin, M.; Ercil, A.; Movellan, J. Data mining spontaneous facial behavior with automatic expression coding. In Lecture Notes in Computer Science; Springer: Berlin/Heidelberg, Germany, 2008; Volume 5042 LNAI, pp. 1-20. [CrossRef]

28. Stratou, G.; Schalk JV, D.; Hoegen, R.; Gratch, J. Refactoring facial expressions: An automatic analysis of natural occurring facial expressions in iterative social dilemma. In Proceedings of the Seventh International Conference on Affective Computing and Intelligent Interaction (ACII), San Antonio, TX, USA, 23-26 October 2017; pp. 427-433.

29. Carrer, S.; Gottman, J.M. Predicting divorce among newlyweds from the first three minutes of a marital conflict discussion. Fam. Process 1999, 38, 293-301. [CrossRef] 
30. Levenson, R.W.; Gottman, J.M. Marital processes predictive of later dissolution: Behavior, physiology, and health. J. Personal. Soc. Psychol. 1992, 63, 221-233.

31. Gottman, J.M.; Notarius, C.I. Decade review: Observing marital interaction. J. Marriage Fam. 2000, 62, 927-947. [CrossRef]

32. Grobova, J.; Colovic, M.; Marjanovic, M.; Njegus, A.; Demire, H.; Anbarjafari, G. Automatic Hidden Sadness Detection Using Micro-Expressions. In Proceedings of the 12th IEEE International Conference on Automatic Face \& Gesture Recognition (FG 2017), Washington, DC, USA, 30 May-3 June 2017. [CrossRef]

33. Guo, J.; Lei, Z.; Wan, J.; Avots, E.; Hajarolasvadi, N.; Knyazev, B.; Kuharenko, A.; Junior, J.C.S.J.; Baró, X.; Demirel, H.; et al. Dominant and Complementary Emotion Recognition from Still Images of Faces. IEEE Access 2018, 6, 26391-26403. [CrossRef]

34. Kulkarni, K.; Corneanu, C.A.; Ofodile, I.; Escalera, S.; Baro, X.; Hyniewska, S.; Allik, J.; Anbarjafari, G. Automatic Recognition of Facial Displays of Unfelt Emotions. J. IEEE Trans. Affect. Comput. 2018. [CrossRef]

35. Noroozi, F.; Marjanovic, M.; Njegus, A.; Escalera, S.; Anbarjafari, G. Audio-Visual Emotion Recognition in Video Clips. IEEE Trans. Affect. Comput. 2017, 10, 26391-26403. [CrossRef]

36. Girard, J.M.; Chu, W.-S.; Jeni, L.A.; Cohn, J.F. Sayette Group Formation Task (GFT) Spontaneous Facial Expression Database. In Proceedings of the 12th IEEE International Conference on Automatic Face and Gesture Recognition (FG), Washington, DC, USA, 30 May-3 June 2017; pp. 581-588.

37. Ringeval, F.; Sonderegger, A.; Sauer, J.; Lalanne, D. Introducing the RECOLA Multimodal Corpus of Remote Collaborative and Affective Interactions. Presented at the Automatic Face and Gesture Recognition (FG), 2013 10th IEEE International Conference and Workshops, Shanghai, China, 22-26 April 2013.

38. Bonnefon, J.-F.; Hopfensitz, A.; Neys, W.D. Can We Detect Cooperators by Looking at Their Face? Curr. Dir. Psychol. Sci. 2017, 26, 276-281. [CrossRef]

39. Brinke, L.T.; Vohs, K.D.; Carney, D.R. Can Ordinary People Detect Deception After All? Trends Cogn. Sci. 2016, 20, 579-588. [CrossRef] [PubMed]

40. Bershadskyy, D.; Othman, E.; Saxen, F. Predicting Free-Riding in a Public Goods Game: Analysis of Content and Dynamic Facial Expressions in Face-to-Face Communication; Publications by Researchers at Halle Institute for Economic Research (IWH), IWH Discussion Papers; Halle Institute for Economic Research (IWH): Halle, Germany, 2019; Available online: http://hdl.handle.net/10419/196589 (accessed on 18 June 2019).

41. Belot, M.; Bhaskar, V.; Ven, J.V.D. Can Observers Predict Trustworthiness? Rev. Econ. Stat. 2012, 94, $246-259$. [CrossRef]

42. Sylwester, K.; Lyons, M.; Buchanan, C.; Nettlea, D.; Roberts, G. The role of Theory of Mind in assessing cooperative intentions. Personal. Individ. Differ. 2012, 52, 113-117. [CrossRef]

43. Bijleveld, C. Fare dodging and the strong arm of the law. J. Exp. Criminol. 2007, 3, 183-199. [CrossRef]

44. Kulkarni, V.; Chapuis, B.; Garbinato, B.; Mahalunkar, A. Addressing the Free-Rider Problem in Public Transport System. arXiv 2018, arXiv:1803.04389.

45. Campion, M.A.; Medsker, G.J.; Higgs, A.C. Relations Between Work Group Characteristics and Effectiveness: Implications for Designing Effective Work Groups. Pers. Psychol. 1993, 46, 823-847. [CrossRef]

46. Bochet, O.; TalbotPage Putterman, L. Communication and punishment in voluntary contribution experiments. J. Econ. Behav. Organ. 2006, 60, 11-26. [CrossRef]

47. Baltrušaitis, T.; Robinson, P.; Morency, L.-P. OpenFace: An open source facial behavior analysis toolkit. In Proceedings of the IEEE Winter Conference on Applications of Computer Vision (WACV), Lake Placid, NY, USA, 7-10 March 2016.

48. Werner, P.; Al-Hamadi, A.; Limbrecht-Ecklundt, K.; Walter, S.; Gruss, S.; Harald, C. Traue Automatic Pain Assessment with Facial Activity Descriptors. IEEE Trans. Affect. Comput. 2017, 8, 286-299. [CrossRef]

49. Saxen, F.; Werner, P.; Al-Hamadi, A. Real vs. Fake Emotion Challenge: Learning to Rank Authenticity from Facial Activity Descriptors. In Proceedings of the IEEE International Conference on Computer Vision Workshops (ICCVW), Venice, Italy, 22-29 October 2017; pp. 3073-3078.

50. Fischbacher, U. Z-Tree: Zurich toolbox for ready-made economic experiments. Exp. Econ. 2007, 10, 171-178. [CrossRef]

51. Bock, O.; Nicklisch, A.; Baetge, I. Hroot: Hamburg registration and organization online tool Olaf. Eur. Econ. Rev. 2014, 71, 117-120. [CrossRef] 
52. Werner, P.; Al-Hamadi, A.; Niese, R.; Walter, S.; Gruss, S.; Traue, H.C. Automatic pain recognition from video and biomedical signals. Presented at the 22nd International Conference on Pattern Recognition, Stockholm, Sweden, 24-28 August 2014.

53. Hughes, G.P. On the Mean Accuracy of Statistical Pattern Recognizers. IEEE Trans. Inf. Theory 1968, 14, 55-63. [CrossRef] article distributed under the terms and conditions of the Creative Commons Attribution (CC BY) license (http://creativecommons.org/licenses/by/4.0/). 